\title{
MYC, Metabolism and Malignancy: Opportunities for Therapeutic Intervention
}

\author{
Achuth Padmanabhan* \\ Department of Molecular Biophysics and Biochemistry, Yale University, USA
}

Metabolic regulation in cells is hugely dependent on the availability of nutrients. Proliferation of cells requires active nutrient uptake, synthesis of nucleic acids, proteins, and lipids and production of ATP. It is thus not surprising those oncogenes that drive cell proliferation also drives metabolic regulation either directly or indirectly. One of the hallmarks of rapidly growing tumor cells is their ability to sustain high rates of glycolysis for ATP generation, irrespective of oxygen availability - a phenomenon known as the Warburg effect $[1,2]$. This shared property of tumor cells provides selective advantage to these cells but also opens up new opportunities for therapeutic intervention. Inhibitors of cellular proliferation that blocks or terminates DNA synthesis or that cross-links or fragments DNA have emerged as some of the classic anticancer agents. These drugs are directed toward normal cellular proliferation processes and therefore lack selectivity for neoplastic cells over proliferating normal cells. The same is true of classic inhibitors of intermediary metabolism, many of which are enzyme transition state analogues.

Since its discovery more than three decades ago as a viral oncogene (v-MYC) in the acutely transforming MC29 virus, MYC has emerged as one of the most commonly deregulated oncogene in human cancers. Apart from being frequently deregulated in many cancers, MYC appears to function downstream of most other oncogenes suggesting that MYC acts a signaling hub and a key downstream effecter. The MYC oncogene, has also been shown to play a major role in cancer cell metabolism. MYC is a basic helix-loop-helix transcription factor that forms a heterodimer with another protein called MAX, and binds DNA upstream of target genes [3]. MYC frequently accumulates in many cancers and has been shown to play a key role in tumor progression [3]. Many recent studies have revealed a strong link between MYC-dependent altered cellular metabolism and tumorigenesis [1,2]. MYC target genes include those involved in the ribosome and mitochondrial biogenesis, regulation of glucose, glutamine and nucleotide metabolism, and DNA replication $[1,2]$. MYC has also been shown to be involved in hypoxic adaptation. The glycolytic genes such as lactate dehydrogenase A and glutaminase are also regulated by MYC through direct and indirect mechanisms. MYC overexpression in cancers, therefore, could concurrently drive aerobic glycolysis and/or oxidative phosphorylation depending on the context of the tumor microenvironment to provide sufficient energy and anabolic substrates for cell growth and proliferation. The dependence of cancer cells on MYC to fulfill their metabolic demands and the role of MYC as a final effector in cancer cells has made it an attractive cancer therapeutic target.

Many studies have suggested that MYC inhibition can halt tumor progression, making it an interesting therapeutic target for new drug development. However, efforts to develop drugs that target MYC have largely proven unsuccessful, partly due to the lack of an obvious target-binding site on the protein. Recently, new approaches have emerged that have shown promise in selectively regulating the function on this elusive cancer target. Rapid and potent abrogation of MYC gene transcription by small molecule inhibitors of the BET family of chromatin adaptors is one such strategy. Disruption of BET bromodomain-promoter interactions leading to subsequent reduction of MYC transcript and protein levels resulted in G1 arrest and extensive apoptosis in a variety of leukemia and lymphoma cell lines [4]. Another emerging strategy is the use of a mutant basic helixloop-helix zipper domain, Omomyc, which dimerizes with MYC and sequesters it in complexes thereby preventing binding of MYC to the E-box. Thus, by acting as a potential dominant negative, Omomyc can reverse tumorigenesis in cancers driven by high-levels of MYC [5]. Small molecules such as 10058-F4, a characterized c-MYC/Max inhibitor, that's targets the MYC/Max interaction has also been shown to lead to cell cycle arrest, apoptosis, and neuronal differentiation [6].

Since MYC acts a major regulator that controls the expression of nearly $15 \%$ of human genes there exists the concern that long-term treatment using the strategies aimed at total loss of MYC function might cause significant and uncontrollable side effects. An alternative strategy would be to use peptides and peptido-mimetics that would specifically interfere with protein-protein interaction between MYC and specific interacting proteins. Although technically challenging, if successful, this strategy would offer the advantage of specifically disrupting specific MYC function without impairing its general role in growth and metabolism. Such a strategy can also be used to fine tune MYC levels in cells. The stability of MYC in cells is regulated by various post-translational modifications. Phosphorylation at different sites influences its turnover and steady state levels $[7,8]$. Thus by designing peptides that would disrupt the interaction of MYC with these specific protein kinases, one could in theory regulate its turnover. This unique ability of his strategy (if successful) to fine tune MYC levels in cells as opposed to a 'complete loss of function' based strategy would help overcome the possible side effects of the later strategy.

\section{References}

1. Dang CV, Le A, Gao P (2009) MYC-induced cancer cell energy metabolism and therapeutic opportunities. Clin Cancer Res 15: 6479-6483.

2. Miller DM, Thomas SD, Islam A, Muench D, Sedoris K (2012) C-Myc and cancer metabolism. Clin Cancer Res 18: 5546-5553.

3. Oster SK, Ho CS, Soucie EL, Penn LZ (2002) The myc oncogene: MarvelousIY Complex. Adv Cancer Res 84: 81-154.

4. Delmore JE, Issa GC, Lemieux ME, Rahl PB, Shi J, et al. (2011) BET bromodomain inhibition as a therapeutic strategy to target c-Myc. Cell 146 904-917.

5. Savino M, Annibali D, Carucci N, Favuzzi E, Cole MD, et al. (2011) The action

*Corresponding author: Achuth Padmanabhan, Department of Molecular Biophysics and Biochemistry, Yale University, New Haven, CT-06511, USA, E-mail: achuth.padmanabhan@yale.edu

Received July 18, 2013; Accepted July 20, 2013; Published July 28, 2013

Citation: Padmanabhan A (2013) MYC, Metabolism and Malignancy: Opportunities for Therapeutic Intervention. J Carcinogene Mutagene 4: 146. doi:10.4172/21572518.1000146

Copyright: (c) 2013 Padmanabhan A. This is an open-access article distributed under the terms of the Creative Commons Attribution License, which permits unrestricted use, distribution, and reproduction in any medium, provided the original author and source are credited. 
Citation: Padmanabhan A (2013) MYC, Metabolism and Malignancy: Opportunities for Therapeutic Intervention. J Carcinogene Mutagene 4: 146. doi:10.4172/2157-2518.1000146

Page of 2

mechanism of the Myc inhibitor termed Omomyc may give clues on how to target Myc for cancer therapy. PLoS One 6: e22284.

6. Huang MJ, Cheng YC, Liu CR, Lin S, Liu HE (2006) A small-molecule c-Myc inhibitor, 10058-F4, induces cell-cycle arrest, apoptosis, and myeloid differentiation of human acute myeloid leukemia. Exp Hematol 34: 1480-1489.

7. Padmanabhan A, Li X, Bieberich CJ (2013) Protein kinase A regulates MYC protein through transcriptional and post-translational mechanisms in a catalytic subunit isoform-specific manner. J Biol Chem 288: 14158-14169.

8. Sears R, Nuckolls F, Haura E, Taya Y, Tamai K, et al. (2000) Multiple Rasdependent phosphorylation pathways regulate Myc protein stability. Genes Dev 14: 2501-2514. 